\title{
Meta-path Hierarchical Heterogeneous Graph Convolution Network for High Potential Scholar Recognition
}

\author{
Yiqing Wu ${ }^{1,2}$, Ying Sun ${ }^{1,2}$, Fuzhen Zhuang ${ }^{1,2 *}$, Deqing Wang $^{3}$, Xiangliang Zhang ${ }^{4}$, Qing $\mathrm{He}^{1,2}$ \\ ${ }^{1}$ Key Lab of Intelligent Information Processing of Chinese Academy of Sciences (CAS), Institute of Computing Technology \\ ${ }^{2}$ University of Chinese Academy of Sciences, Beijing 100049, China \\ ${ }^{3}$ School of Computer Science and Engineering, Beihang University, China \\ ${ }^{4}$ King Abdullah University of Science and Technology, Thuwal, Saudi Arabia \\ zhuangfuzhen@ict.ac.cn, *Corresponding author.
}

\begin{abstract}
Recognizing high potential scholars has become an important problem in recent years. However, conventional scholar evaluating methods based on hand-crafted metrics can not profile the scholars in a dynamic and comprehensive way. With the development of online academic databases, large-scale academic activity data become available, which implies detailed information on the scholars' achievement and academic activities. Inspired by the recent success of deep graph neural networks (GNNs), we propose a novel solution to recognize high potential scholars on the dynamic heterogeneous academic network. Specifically, we propose a novel Mate-path Hierarchical Heterogeneous Graph Convolution Network (MHHGCN) to effectively model the heterogeneous graph information. MHHGCN hierarchically aggregates entity and relational information on a set of metapaths, and can alleviate the information loss problem in the previous heterogenous GNN models. Then to capture the dynamic scholar feature, we combine MHHGCN with Long Short Term Memory (LSTM) network with attention mechanism to model the temporal information and predict the potential scholar. Extensive experimental results on real-world high potential scholar data demonstrate the effectiveness of our approach. Moreover, the model shows high interpretability by visualization of the attention layers.
\end{abstract}

Index Terms-High Potential Scholar, Graph Neural Network, Heterogeneous Graph

\section{INTRODUCTION}

High potential scholars are researchers who are likely to make big scientific achievements in the future. Recognizing high potential scholars is an important problem that always attracts researchers' attention, which can bring benefits in many perspectives. At the national level, recognizing high potential scholars can help the government to better allocate the resources to be invested to the researchers. At the organization level, high potential scholars are treasured human resources in a research institution, finding high potential scholars and attaching more importance to their development helps the institutions to build a higher-level expert group. And at the individual level, recognizing high potential scholars helps the researchers to track works that may be influential in the future.

Traditionally, many metrics can evaluate the achievement of a scholar. For example, the citation count, h-index [1], number of publications, etc. However, it is difficult to recognize high potential scholars with these metrics. Since these metrics can only statically profile the current achievement of scholars, while failing to measure a scholar's potential in a dynamic perspective. Indeed, high potential scholars are not necessarily standing out among peers at the current time, they are likely to improve their academic influence speedily in the future.

Fortunately, with the development of web services, largescale datasets of scholar activities become available. For example Google Scholar, and Microsoft Academic. These online datasets collect academic publication and citation behaviors that happen every day, which naturally form a dynamic heterogeneous academic network. In this network, entities like authors, papers, and conferences are connected by behaviours such as writing, citing, and co-author behaviours. Compared with previous statistical measurements, the academic network not only implies more detailed information of a scholar's achievement but also has a dynamic nature. It provides an unparalleled opportunity to recognize high potential scholars in a dynamic and comprehensive manner.

In recent years, graph deep learning models have made great progress. Graph Neural Network (GNN) is a powerful deep representation learning method for graph data and has got a series of new state-of-the-art benchmarks in various network representation learning tasks [2] [3], And GNN has been widely used for node classification task. . For example, Ye et al. utilized GCN to find talented employees on an inter-company communication network [4], while it designed for homogeneous networks, and thus can't well model the information on the heterogeneous academic network.

Indeed, recognizing high potential scholars on the heterogeneous academic network is far from an easy task. some previous works use GCNs on heterogeneous graph classification, they typically abandon heterogeneous information to transform the heterogeneous graph into a homogeneous graph. For example, some works equally treat different types of nodes and relations. Some works (e.g., HAN [5]) only consider the end nodes of a predefined meta-path on the graph, ignoring 
the internal node and relational information. However, the heterogeneous information is essential for comprehensively measuring the potential of scholars, which cause these methods not suitable for this task.

In this paper, we propose a novel solution to recognize high potential scholars on a heterogeneous academic graph. Specifically, we first form a dynamic heterogeneous academic graph, and predict high potential scholars with both GCN and dynamic modeling in a comprehensive way. Specially, we propose a novel Mate-path Hierarchical Heterogeneous Graph Convolution Network (MHHGCN) for feature extraction on the academic graph. MHHGCN hierarchically aggregates features of node based on a set of meta-path and combines the meta-path-specific features with attention mechanism for the final classification, the structure can alleviate the information loss problem in the previous works. We conduct experiments with real-world publication data collected from an online database, the experimental results demonstrate our model not only significantly outperforms the baseline models but also provides interesting insights by visualizing and analyzing on the attention layers.

The major contributions of this paper can be summarized as follows:

- We profile scholar in a dynamic heterogeneous network, which contains more information than the homogeneous or static graphs.

- We propose a novel Mate-path Hierarchical Heterogeneous Graph Convolution Network (MHHGCN), which captures different semantics information in the heterogeneous graph and achieves more accurate heterogeneous feature extraction, by making use of the internal relations contained in meta-path.

- We evaluate our approach with extensive experiments on the real-world publication data. The results reveal interesting insights and validate the effectiveness of our approach in terms of high potential scholar recognition.

\section{Preliminaries}

In this section, we introduce concepts of the heterogeneous graph and formulate the problem.

\section{A. Heterogenous Graph}

Definition 2.2. Heterogeneous Graph [6] A heterogeneous graph is defined as a directed graph $G=(V, E)$, which consists of a node set $V$ and edge set $E$. A heterogeneous graph has an node type mapping function $\phi(v): V \rightarrow \mathcal{A}$ and a edge type mapping function $\psi: E \rightarrow \mathcal{R}$. $\mathcal{A}$ and $\mathcal{R}$ denote the sets of predefined node types and edge types, where $|\mathcal{A}|+|\mathcal{R}|>2$.

Definition 2.3 Meta-Path [7] A meta-path $\mathrm{P}$ is defined as a path in the form of $p_{1} \stackrel{\mathbf{r}_{1}}{\longrightarrow} p 2 \stackrel{\mathbf{r}_{2}}{\longrightarrow} \ldots \stackrel{\mathbf{r}_{l}}{\longrightarrow} p_{l+1}$ (abbreviated as $p_{1} p_{2} \ldots p_{l+1}$ ), which denotes a composite relation $R=\mathbf{r}_{1}$ 。 $\mathbf{r}_{2} \circ \ldots \circ \mathbf{r}_{l}$ between node types $p_{1}$ and $p_{l}$, where $\circ$ denotes the composition operator on relations. What more we define $P^{-1}=p_{l} \ldots p_{2} p_{1}$ and $R^{-1}=\mathbf{r}_{l}^{-1} \circ \ldots \circ \mathbf{r}_{2}^{-1} \circ \mathbf{r}_{1}^{-1}$ as the transpose of $P$ and $R$ respectively.
Definition 2.4 Meta-path based Neighbors. Given a node $v$ and a meta-path $P$ in a heterogeneous graph, the meta-path based neighbors $N_{v}^{P}$ of node $v$ are defined as the set of nodes that can reach node $v$ by the meta-path $P$. It is notable that $v \in N_{v}^{P}$.

\section{B. Problem Formulation}

Our real-world high potential scholar data contains two parts. The first part is yearly organizational academic data crawled from the Microsoft Academic ${ }^{1}$ and can be regarded as a heterogenous academic network. On this academic network, nodes represent different kinds of academic entities (e.g., scholar and paper), and edges represent different relationships between the entities (e.g., write and cite). The second part is the high potential scholar list, which comes from the National Natural Science Foundation of China (NSFC) ${ }^{2}$ called the National Science Fund for Excellent Young Scholars (EYS).

Formally, we use $G^{S P}=<V, E, \phi>$ to denote an academic network, where $V$ denotes the set of entities, $E$ denotes a set of directed edges, and $\phi(\cdot)$ denotes the node type indicator. Specifically, $\phi\left(v_{s}\right)=t_{S}$ when $v_{s}$ is a scholar node, and $\phi\left(v_{p}\right)=t_{P}$ when $v_{p}$ is a paper node. Besides we use $\xi(v): v_{s} \rightarrow \mathcal{X}_{S}, v_{p} \rightarrow \mathcal{X}_{P}$ to represent the mapping function that maps each type of nodes to their feature vectors. we also define several kinds of edge set. Specifically, an edge in $E_{w}=\left\{e_{u_{s} v_{p}} \mid e_{u_{s} v_{p}} \in E, \phi\left(v_{s}\right)=t_{S}, \phi\left(v_{p}\right)=t_{P}\right\}$ represents that $v_{s}$ wrote $v_{p}$ (i.e., $v_{p}$ was written by $v_{s}$ ). And an edge in $E_{c}=\left\{e_{u_{p} u_{p}^{\prime}} \mid e_{u_{p} u_{p}^{\prime}} \in E, \phi\left(u_{p}\right)=t_{P}, \phi\left(u_{p}^{\prime}\right)=t_{P}\right\}$ represents that $v_{p}$ cited $v_{p}^{\prime}$ (i.e., $v_{p}^{\prime}$ was cited by $v_{p}$ ).

Academic networks from time period $t$ to time period $t+K$ form a sequence of network $G_{t: t+K}^{S P}=$ $\left\{G_{t, t+1}^{S P}, G_{t, t+2}^{S P}, \ldots, G_{t, t+K}^{S P}\right\}$, where $G_{i: i+k}^{S P}$ represents an academic network that contains academic activities from time period $i$ to time period $i+k$. Given this sequence of heterogeneous academic networks, we aim to develop a classification model $f\left(v_{s}, G_{i: i+k}^{S P}\right)=y$ to predict whether a scholar $v_{s}$ is a high potential scholar. In this paper, we label the scholars by observing whether they will get the afore-mentioned funding awards in the future $m$ years and train the classification model with a supervised-learning-based methodology.

\section{High Potential Scholar Recognition}

In this section, we introduce technical details of our high potential scholar recognition model.

\section{A. Overall Structure}

The academic network of a scholar is fast-changing, especially for young scholars, which means the fast-changing scholar profile. So we need to model scholars in a dynamic academic network to capture the change of scholar profile. Similar to the work proposed by Ye et al. [4], we separate the dynamic academic network into a sequence of static academic networks. Considering the complexity and rationality, we adopt a sequence of year-level academic networks.

\footnotetext{
${ }^{1}$ https://academic.microsoft.com/

${ }^{2}$ http://www.nsfc.gov.cn/
} 
For each academic network, we first project each type of node features to the same feature space. Then we extract the node embeddings with Meta-path Hierarchical Heterogeneous Graph Convolutional Network (MHHGCN). Finally, we feed sequential learned scholar representations into Recurrent Neural Networks (RNN) with attention mechanism to get final scholar profile representation and predict the scholar potential.Fig. 1 shows the overall structure of our method.

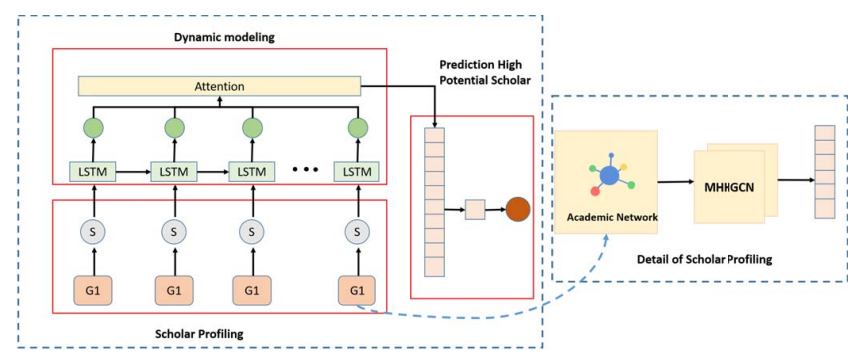

Fig. 1. Overall structure of high potential scholar recognition model.

\section{B. Heterogenous Graph Feature Extraction}

To extract graph information from each heterogeneous academic graph, we propose a novel Hierarchical Heterogeneous Graph Convolution Network (HHGCN). MHHGCN uses a meta-path-based hierarchical structure to separately extract node embeddings with different semantics and aggregates them into a unified representation. Specifically, firstly, to better model the information implied in a meta-path, MHHGCN hierarchically aggregates node information in the meta-path to learn the meta-path-specific embeddings of the target nodes. Then the semantic aggregator combines different meta-pathspecific embeddings with an attention mechanism. The details of MHHGCN will be introduced in the next section.

\section{Dynamic Scholar Potential Prediction}

With MHHGCN, we can obtain a sequence of scholar profiles by modeling the static academic networks. Then we use Long Short-term Memory (LSTM) [8] network, to capture the change of scholar profile. Specially, the input of LSTM a sequence of embeddings of a scholar $z=\left\{z_{1}, \ldots, z_{k}\right\}$. For each step $t$ with the input scholar embedding $z_{t}$ and hidden state vector $h_{t-1}$, LSTM computes the updated hidden state vector $h_{t}=\operatorname{LSTM}\left(h_{t-1}, z_{t}\right)$. Finally LSTM outputs a sequence of hidden representation $\left\{h_{1}, h_{2}, \ldots, h_{k}\right\}$.

Considering that different year-level embeddings have different contributions to final scholar embedding, we adopt a year-level attention mechanism to measure the importance. Specifically, given a sequence of year-level embeddings $h_{1}, h_{2}, \ldots, h_{k}$ of scholar $v_{s}$, the year-level attention mechanism can be formulated as following:

$$
\begin{aligned}
& e_{v_{s}}^{j}=q^{\top} \cdot h_{j}, \quad a_{v_{s}}^{j}=\frac{\exp \left(e_{v_{s}}^{j}\right)}{\sum_{k=1}^{K} \exp \left(e_{v_{s}}^{k}\right)}, \\
& z_{v_{s}}^{\prime}=\sum_{k}^{K} a_{v_{s}}^{k} \cdot h_{j},
\end{aligned}
$$

where $q^{\top}$ is the attention vector, $z_{v_{s}}^{\prime}$ is the final learned embedding of the scholar $v_{s}$.

Finally, we apply a fully-connected network with a sigmoid function to learn a one-dimension vector $d$ for predicting the label of a scholar, formulated as:

$$
y=\operatorname{sigmod}\left(W_{\text {pre }} \cdot z_{v_{s}}^{\prime}+b_{\text {pre }}\right),
$$

where $y$ is the output of our model. Here we use Cross-Entropy Loss as the loss function of our model.

\section{Meta-Path Hierarchical Heterogenous Graph CONVOlutional NeTWORK}

In this section, we introduce the detailed structure of MHHGCN. The structure of MHHGCN is shown in Fig. 2. Specifically, we separately introduce the major components of MHHGCN, including node feature projection and hierarchical meta-path aggregation layers.

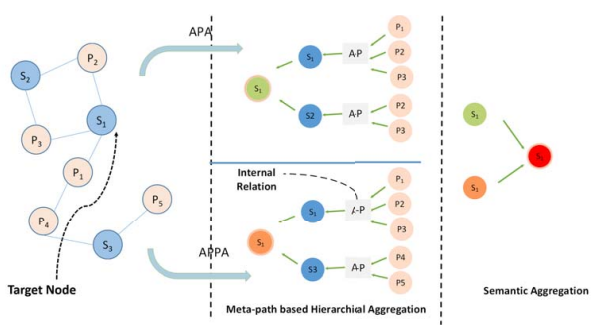

Fig. 2. Overall the architecture of MHHGCN

\section{A. Node Feature Projection}

Before aggregating the nodes' information, we should notice that for a heterogeneous graph, different types of nodes have different feature spaces. So we design a type-specific transformer for each type of node, which projects each node's feature into the same feature space. For a node $v \in V_{t}$ of type $t \in T$, the project function can be shown as follows:

$$
h_{v}=M_{t} \cdot x_{v},
$$

where $x_{v}$ and $h_{v}$ are the initial feature and projected feature respectively. $M_{t} \in \mathbb{R}^{d \times d^{\prime}}$ is the type-specific transform matrix.

\section{B. Internal Relation Aggregation}

Although a meta-path reflects the relation between two end nodes, the internal relations in meta-path also contain some important information, while most existing meta-path based GCNs drop that information. To utilize those information, we propose a hierarchical aggregation, which contains two parts: internal relation aggregation and meta-path aggregation.

The key idea of internal relation aggregation is that the internal relations can be used to learn better representations of two end type nodes of a meta-path, which contains more graph structure and node information that helps meta-path aggregation aggregate meta-path based neighbors to target nodes more effectively. 
Specifically, consider a long meta-path with complicated internal relations, the selection of aggregated internal relations will influence the effectiveness of the model. Intuitively, the closer a node is to $v$, the more relevant it is to $v$. Based on this assumption, we define $k$ meta-path internal relations for different meta-path.

Definition $k$ Meta-path Internal Relations: Given a metapath $P\left(p_{1} p_{2} \ldots p_{l}\right)$ of length $l$ and a receptive field $k$. the $\mathrm{k}$ meta-path internal relations is defined as a set of relations: $R_{k}^{P}=\left\{r_{1}^{P}, r_{2}^{P}, \ldots, r_{k}^{P}\right\} \cup\left\{r_{1}^{P^{-1}}, r_{2}^{P^{-1}}, \ldots, r_{k}^{P^{-1}}\right\}$, where $r_{i}^{P}$ denotes the relation between $p_{1}$ and $p_{1+i}$ (i.e., $p_{1} p_{2} . . p_{1+i}$ ) and $r_{i}^{P^{-1}}$ denotes the relation between $p_{l}$ and $p_{l-i}$ (i.e., $\left.p_{l} p_{l-1} \ldots p_{l-i}\right)$. In addition, similar with meta-path based neighbors, we use $N_{v}^{r_{i}^{P}}$ to denote the relation $r_{i}^{P}$ based neighbors of node $v$.

Intuitively, for an internal relation $r$, different nodes in $N_{v}^{r}$ may have different importance for the target node $v$. For this consideration, we adopt attention mechanism to learn the contribution degree of $r$ based neighbors. Specially, given a node $v$ and it's relation $r$ based neighbors node $u \in N_{v}^{r}$, the node-level attention can learn the factor $e_{v, u}^{r}$, meaning the importance of node $u$ for node $v$. Here we adopt self-attention on nodes as attention function, which formulated by following:

$$
\begin{aligned}
& e_{v, u}^{r}=\operatorname{LeakyReLU}\left(a_{r}^{\top} \cdot\left[h_{v} \| h_{u}\right]\right), \\
& a_{v, u}^{r}=\frac{\exp \left(e_{v, u}^{r}\right)}{\sum_{w \in N_{v}^{r}} \exp \left(e_{v, w}^{r}\right)}, \quad h_{v}^{r}=\sigma\left(\sum_{w \in N_{v}^{r}} a_{v, w}^{r} \cdot h_{w}\right),
\end{aligned}
$$

where $a_{r}^{\top} \in \mathbb{R}^{2 d^{\prime}}$ is a learn-able attention vector for metapath $P$ or internal-relation $r$ of meta-path $P . a_{v, u}^{p}$ is the normalized attention coefficient, which is used to computer new embedding $h_{v}^{r} \cdot \sigma(\cdot)$ is activation function, we adopt $R E L U$ function in our paper.

However, heterogeneous graphs are too complicated, which makes a high variance of graph data. To solve this problem, we extend the attention mechanism to multi-head attention, which helps the learning process to be more stable. So for each aggregator we use $\mathrm{K}$ independent self-attention head, and concatenate the learned embeddings as the output, which is formulated as following:

$$
z_{v}^{r}=\|_{k=1}^{K}\left(h_{v}^{r}\right)
$$

Here we use the attention mechanism described above as the aggregator. Formally given a relation $r$, a node $v$ with feature $h_{v}$ and it's $r$ based neighbors $u \in N_{v}^{r}$ with node feature $h_{u}$, the aggregator can be formulated as:

$$
z_{v}^{r}=A G G R E G A T O R^{r}\left(h_{v},\left\{h_{u}, \mid \forall u \in N_{v}^{r}\right\}\right) .
$$

Now given a meta-path $P$, which starts with node type $A$ and end with type $B$, and a set of internal relations $R_{k}^{P}=$ $\left\{\left\{r_{1}^{P}, r_{2}^{P}, \ldots, r_{k}^{P}\right\},\left\{r_{1}^{P^{-1}}, r_{2}^{P^{-1}}, \ldots, r_{k}^{P^{-1}}\right\}\right\}$, we first apply the relation-specially aggregator to each relation and get a series of embedding groups $\left\{Z_{1}^{P}, Z_{2}^{P}, \ldots, Z_{k}^{P}, Z_{1}^{P^{-1}}, Z_{2}^{P^{-1}}, \ldots, Z_{k}^{P^{-1}}\right\}$, then we connect the learned embeddings with the end types nodes' projected embeddings and generate new embeddings $H_{A}^{P}$ and $H_{B}^{P}$, which are the target embeddings of our internal relation aggregation.

\section{Meta-path Aggregation}

After internal relation aggregation for a meta-path $P$, we calculate new embeddings of two end nodes, which will be used to our meta-path aggregation. Similar to internal aggregation, for a node $v$, its meta-path based neighbors show different importance in learning node embeddings. So here we use the same aggregator defined in the internal aggregation section to aggregate information. Specifically, given a node $v$ and it's meta-path $P$ based neighbors $u \in N_{v}^{P}$. The meta-path specially aggregator can be described as following:

$$
z_{v}^{P}=\operatorname{AGGREGATOR}{ }^{P}\left(h_{v}^{P}, \mid\left\{h_{u}^{P}, \mid \forall u \in N_{v}^{P}\right\}\right),
$$

where $h_{v}^{P}$ and $h_{u}^{P}$ are the embedding learned by internal aggregation, $A G G R E G A T O R^{P}$ is the meta-path specific aggregator $z_{v}^{P}$ is the meta-path-specific embedding .

\section{Semantic Aggregation}

Different meta-path reflects different semantic information and shows different importance for a specific task. Based on this idea, we propose a semantic aggregator based on the attention mechanism. For node $v \in V_{A}$, where $A$ denotes the node type, we feed $M$ meta-path-specific node embeddings $\left\{z_{v}^{P_{0}}, z_{v}^{P_{1}}, \ldots, z_{v}^{P_{M}}\right\}$ to semantic aggregator to learn the graph embedding $z_{v}$. The attention mechanism can be formulated as:

$$
\begin{aligned}
& e_{v}^{P_{k}}=q_{A}^{T} \cdot \tanh \left(W_{A} \cdot z_{v}^{P_{k}}+b\right), \\
& a_{v}^{P_{k}}=\frac{\exp \left(e_{v}^{P_{k}}\right)}{\sum_{k \in M} \exp \left(e_{v}^{P_{k}}\right)}, z_{v}=\sigma\left(\sum_{k=1}^{M} a_{v}^{P_{k}} \cdot z_{v}^{P_{k}}\right)
\end{aligned}
$$

where $W_{A}$ is a learnable transform matrix, $q_{A}^{T}$ is the parameterized attention vector, $e_{v}^{P_{k}}$ is the importance of meta-path $P_{k}$ for node $v, a_{v}^{P_{k}}$ is the normalized importance of meta-path, which is used to computer final embedding $z_{v}$ of a heterogeneous graph for node $v$. Specially, $z_{v}$ is scholar's profile in academic network.

\section{EXPERIMENTS}

In this section, we will evaluate our proposed model based on the large-scale real-world dataset.

\section{A. Experimental Setups}

1) Data Preprocessing: As introduced in Section 2, the two sub-datasets academic network and EYS In the EYS, there are 9 year-level academic networks from 2006 to 2015, including 1,003,824 scholars and 4,063,040 papers. We select the EYS awardees in the field of Computer and Information Science and Engineering as positive samples, and randomly select some scholars who have similar research age with the awardees as negative samples. For each scholar, we use successive 6 yearlevel academic and networks to predict if he/she will get the 
award in the future 5 years. Finally, for EYS we have 692 positive samples, 792 negative samples, In this paper, we use meta-path $A P A, A P P A$ to predict high potential scholars, which mean the co-author and citation relation between two scholars respectively. What's more we set receptive filed of $k$ meta-path internal relations to 1 . To accelerate the training, we extract ego-academic networks for the samples. Specifically, an ego-academic network is centered on the target scholar and contains 1) the 2-hop author meta-path neighbors based on meta-path $A P A, 2)$ the 1-hop author meta-path neighbors based on $A P P A$, and 3 ) the selected nodes' papers. To limit the scale of the network, for each author found by $A P A$, we only retain their top-19 most frequent co-authors.

For each scholar and paper in the academic network, we extract some features to increase the model's fitting ability. We use the citation count publication count and the average of paper's citation count at the end of year $i$, as the feature of a scholar, named as a content feature. Then we extract social centrality scores from the co-author network. Inspired by the work of [9] [10], we extract PageRank [11], Hubs and Authorities [12], constraint [13], Degree centrality, closeness centrality [14], eigenvector centrality [15]. For a paper, we calculate the average citation count of it since it published, the last five years publication count, citation count and average paper citation count of the venue or journal as the features.

2) Baselines: We compare our model with two categories of baseline methods, which are Non-GNN models and graph neural networks based models. We also do an ablation experiment to show the effectiveness of our model. Specifically, we have the following baseline models:

- Support Vector Machine (SVM) [16]: SVM is a classic supervised model for the classification tasks. We connect all years features and node centralities of a scholar as the input. Here we use the linear kernel.

- Logic Regression (LR): a classic model for classification tasks, we use the same features with SVM.

- LSTM [8]: a neural network for sequential data modeling, where we use the dynamic author features as input.

- GCN [17]: A GNN designed for homogeneous graphs. Here we test all the meta-path and report the best one.

- GAT [3]: A attention-based GNN designed for homogeneous graphs. Here we test all the meta-path for GAT and report the best one.

- HAN [5]: A GNN designed for heterogeneous graph, which is a meta-path based model. To be fair, here we select the same meta-path with our model.

- MHHGCNnh: It is a variant of our framework, which removes the meta-path hierarchical aggregation, only uses the meta-path based neighbors. Here we regrade the internal relation as a simple mete-path and adopt APPA, APA, and AP as meta-path.

3) Network Configurations: For GCN and GAT we use two-layer graph convolution. For GAT, HAN, and our proposed MHHGCN, we set the dropout rate to 0.2. For all models, we use learning rate 0.005 , optimize model with
Adam [17],. Here we use early stopping with the patience of 15 . We set the batch size to 120 .

\section{B. Evaluation on High Potential Scholar Recognition}

Here we use five-fold cross validation, where for each fold we randomly selected $80 \%$ as training data, $20 \%$ as test data. What's more considering the high various of graph data, for the GNN based models, we run GNN models five times and report the averaged performance. The results are shown in table I.

We have several observations. First, our model outperforms the baseline models both in AUC and F1, which proves the effectiveness of our proposed framework. Second, the sequential models (i.e., LSTM) outperforms classic models. This result proves the necessity of dynamic scholar profiling for high potential scholar recognition. Third, GCN performs worst than LSTM, we attribute this to GCN simply averages neighbors' information while unable learns the importance of neighbors and will import noise. Fourth, the GCN for heterogeneous graph performs better than GCN for homogeneous graph, which is because heterogeneous graphs have more information and can help to profile scholar in a comprehensive way. Fifth, our MHHGCN performs better than HAN when using the same meta-path, which is because we make use of the internal relations and nodes information of meta-path. Sixth, while MHHGCNnd uses all types of nodes information (paper and author) and same relations (AP, APPA, and APA), the performance decreases a lot when disabling the hierarchical aggregator. This result shows that the hierarchical aggregator learns better scholar representations than simply combining the node information and relations.

TABLE I

PREDICTION PERFORMANCE OF DIFFERENT METHODS

\begin{tabular}{ccccc}
\hline Model & AUC & Prec. & Rec. & F1. \\
\hline \hline LR & 79.85 & 72.50 & 65.06 & 68.50 \\
SVM & 71.52 & 71.90 & 66.59 & 69.08 \\
LSTM & 81.86 & 73.79 & 70.01 & 71.51 \\
GCN & 81.52 & 70.69 & 71.61 & 70.65 \\
GAT & 83.33 & $\mathbf{8 4 . 0 1}$ & 70.02 & 76.3 \\
HAN & 85.57 & 81.6 & 73.16 & 76.95 \\
MHHGCN $_{n d}$ & 84.44 & 79.97 & 71.83 & 75.48 \\
MHHGCN & $\mathbf{8 7 . 7 8}$ & 81.56 & $\mathbf{7 6 . 4 9}$ & $\mathbf{7 8 . 7 4}$ \\
\hline
\end{tabular}

\section{Attention Analysis}

In our paper, we use attention mechanism to estimate the importance of nodes, meta-path, and year-level scholar profiles. To get more insights of our model, we make a detailed analysis of our three attention mechanisms.

Analysis of node attention. In MHHGCN, there is an attention layer that calculates the importance of the nodes' relation or meta-path based neighbors. In Fig. 3(a), we show 4 APA based neighbors of scholar A1 who have the highest attention values. By analyzing the academic network, If we sort A1's co-authors by their co-author frequency with A1, 
we found that $\mathrm{A} 2$ and $\mathrm{A} 3$ are the top- 1 and top- 2 co-author of A1. This means our attention layer learns which co-author is more important. The scholar with the third highest attention value is A1 itself, Indeed, a scholar's own feature is important for potential evaluation.
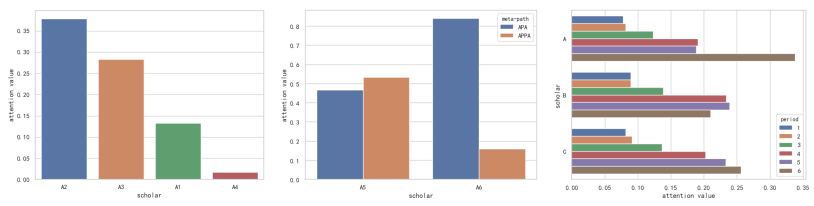

(a) Attention value of (b) Attention value of (c) Attention value of A1's neighbors different meta-path each period

Fig. 3. Attention Analysis

Analysis of meta-path attention The semantic aggregator in MHHGCN learns the importance of each meta-path for a specific-scholar. To show the effect of semantic aggregator. We visualize the meta-paths attention value for some scholars in Fig. 3(b). We can observe that the importance of different meta-path differs for these scholars. Specifically, meta-path APPA is more important for profiling A5, while meta-path APA is more important for scholar A6. By analyzing our data, we find that scholar A5 published 7 papers and received 494 citations this year, A6 also published 7 papers and received 364 citations in this year. But we notice that the 7 papers of A7 include 2 publications in ECCV and 1 publication in CVPR, which are known as the top venue of computer vision filed. Since APA path is related to publishing activities and APPA is related to citation activities, we can observe that this importance allocation makes sense. This also implies that MHHGCN has the ability of distinguishing the quality of papers to better profile the scholars.

Analysis of LSTM attention MHHGCN uses LSTM with attention to obtain the contributes of each year-level scholar profile to finally high potential scholar prediction. Here we randomly select three scholars and visualized the year-level attention of them. As is shown in Fig. 3(c), different scholars have different attention values in different periods, which verifies our assumption that dynamic profiling is essential for recognizing the high potential scholars. Generally, the closer a year is to the current year, the more important the year-level scholar profile is. Indeed, the academic achievement in recent years is important to decide if someone is a high potential scholar.

\section{CONCLUSION}

In this paper, we proposed a novel solution to recognize high potential scholars on the dynamic heterogeneous academic network. Specifically, we proposed a novel Matepath Hierarchical Heterogeneous Graph Convolution Network (MHHGCN) to effectively model the heterogeneous graph information. MHHGCN hierarchically aggregates entity and relational information on a set of meta-paths. Then to capture the dynamic scholar feature, we combined MHHGCN with Long Short Term Memory (LSTM) network with attention mechanism to model the temporal information and predict the scholar potential. Finally, we conducted extensive experiments on a real-world dataset, which demonstrated the effectiveness of our approach. Moreover, the model shows high interpretability by visualization of the attention layers.

\section{ACKNOWLEDGEMENTS}

The research work is supported by the National Key Research and Development Program of China under Grant No. 2018YFB1004300, the National Natural Science Foundation of China under Grant NOs. U1811461, 61773361, U1836206, the Project of Youth Innovation Promotion Association CAS under Grant No. 2017146.

\section{REFERENCES}

[1] J. E. Hirsch, "An index to quantify an individual's scientific research output," Proceedings of the National academy of Sciences, vol. 102, no. 46, pp. 16569-16572, 2005.

[2] W. Hamilton, Z. Ying, and J. Leskovec, "Inductive representation learning on large graphs," in Advances in neural information processing systems, 2017, pp. 1024-1034.

[3] P. Veličković, G. Cucurull, A. Casanova, A. Romero, P. Lio, and Y. Bengio, "Graph attention networks," arXiv preprint arXiv:1710.10903, 2017.

[4] Y. Ye, H. Zhu, T. Xu, F. Zhuang, R. Yu, and H. Xiong, "Identifying high potential talent: A neural network based dynamic social profiling approach," in 2019 IEEE International Conference on Data Mining (ICDM). IEEE, 2019, pp. 718-727.

[5] X. Wang, H. Ji, C. Shi, B. Wang, Y. Ye, P. Cui, and P. S. Yu, "Heterogeneous graph attention network," in The World Wide Web Conference, 2019, pp. 2022-2032.

[6] Y. Sun and J. Han, "Mining heterogeneous information networks: a structural analysis approach," Acm Sigkdd Explorations Newsletter, vol. 14, no. 2, pp. 20-28, 2013.

[7] Y. Sun, J. Han, X. Yan, P. S. Yu, and T. Wu, "Pathsim: Meta pathbased top-k similarity search in heterogeneous information networks," Proceedings of the VLDB Endowment, vol. 4, no. 11, pp. 992-1003, 2011.

[8] S. Hochreiter and J. Schmidhuber, "Long short-term memory," Neural computation, vol. 9, no. 8, pp. 1735-1780, 1997.

[9] H. Huang, Y. Dong, J. Tang, H. Yang, N. V. Chawla, and X. Fu, "Will triadic closure strengthen ties in social networks?" ACM Transactions on Knowledge Discovery from Data (TKDD), vol. 12, no. 3, pp. 1-25, 2018.

[10] G. Farnadi, J. Tang, M. De Cock, and M.-F. Moens, "User profiling through deep multimodal fusion," in Proceedings of the Eleventh ACM International Conference on Web Search and Data Mining, 2018, pp. 171-179.

[11] L. Page, S. Brin, R. Motwani, and T. Winograd, "The pagerank citation ranking: Bringing order to the web." Stanford InfoLab, Tech. Rep., 1999.

[12] S. Chakrabarti, B. E. Dom, S. R. Kumar, P. Raghavan, S. Rajagopalan, A. Tomkins, D. Gibson, and J. Kleinberg, "Mining the web's link structure," Computer, vol. 32, no. 8, pp. 60-67, 1999.

[13] R. S. Burt, Structural holes: The social structure of competition. Harvard university press, 2009.

[14] A. Bavelas, "Communication patterns in task-oriented groups," The journal of the acoustical society of America, vol. 22, no. 6, pp. 725-730, 1950.

[15] P. Bonacich, "Power and centrality: A family of measures," American journal of sociology, vol. 92, no. 5, pp. 1170-1182, 1987.

[16] R.-E. Fan, K.-W. Chang, C.-J. Hsieh, X.-R. Wang, and C.-J. Lin, "Liblinear: A library for large linear classification," Journal of machine learning research, vol. 9, no. Aug, pp. 1871-1874, 2008.

[17] T. N. Kipf and M. Welling, "Semi-supervised classification with graph convolutional networks," arXiv preprint arXiv:1609.02907, 2016. 